\title{
An Analysis of Spaces of Range Image Small Patches
}

\author{
Qingli Yin ${ }^{1, *}$ and Wen Wang, ${ }^{2, *}$ \\ ${ }^{1}$ Department of Laboratory and Facility Management, Shandong Jianzhu University, Jinan 250101, P.R. China; ${ }^{2}$ School \\ of Science, Shandong Jianzhu University, Jinan 250101, P.R. China
}

\begin{abstract}
In the paper some topological properties of spaces of range images are studied partially. The methods of computing topology are used into the spaces of $4 \times 4$ and $6 \times 6$ high contrast blocks, the following result is obtained: both of the high contrast blocks spaces have a subspace that is topologically equivalent to the Klein bottle, which indicates the local behavior of optical image blocks and range image blocks perhaps are very similar.
\end{abstract}

Keywords: High-contrast patches, klein bottle, persistent homology, range images, topology.

\section{INTRODUCTION}

In the recent years, a lot of studies about image statistics were carried out [1-3]. Some satisfactory results about the topic have been obtained from regions for example, neuroscience and physiology to computer vision. In this paper we will analyze the structure of high-contrast regions of range images instead of looking at an image as a whole. The greatest advantage of analyzing range images locally is that reduces the dimensionality of the problem. A. Lee, K. Pedersen, and D. Mumford [3] research distributions of $3 \times 3$ blocks (or patches) of optical images and range images, they observed that high-contrast $3 \times 3$ range blocks are thickly gathered round the binary patches and the majority of the high-contrast optical blocks locate close to a twodimensional circular ring. Instead of studying statistical behaviors of these blocks, we are concern about the homology of the space of $n \times n$ high contrast blocks with enough little $n$. The authors of the paper [4] use topological instruments into the dataset of optical image blocks, they discover a tall density subset known as the primary circle, and certify that there exists a large 2-dimensional subset having the homology of a Klein bottle which includes the primary circle. In [5], Adams and Carlsson shown that $5 \times 5$ and $7 \times 7$ range patches have the primary circle behavior.

In the paper, we use the techniques in the paper [4] to high-contrast regions of range images, and study the topological structure of spaces of $4 \times 4$ and $6 \times 6$ range patches. In particular, we prove that there exists a 2-dimensional subspace in each of the two spaces, of which homology is that of a Klein bottle.

\section{PERSISTENT HOMOLOGY}

For a finite point set $X$ sampled from an underlying space $X \subseteq R^{m}$, together with a parameter $\varepsilon$, one can build from it a simplicial complex, called the Vietoris-Rips, indicated $V R_{\varepsilon}$. The vertex set of this complex is $X$, and a set $\left\{y_{0}, y_{1}, \ldots, y_{p}\right\}$ determine a $p$-simplex in $V R_{\varepsilon}$ iff $d\left(y_{i}, y_{j}\right) \leq \varepsilon$ for all $0 \leq i, j \leq$ $p$. Here $d$ is the metric distance. One can compute a $V R_{\varepsilon}$ complex at each scale $\varepsilon$. Obviously, $V R_{0}=\varnothing$ and $V R_{\infty}$ is an (| $X \mid-1)$-simplex. That means, the $V R_{\varepsilon}$ complex may have a much higher dimension than the embedding space $X$. But, there exists a proper scope whence $V R_{\varepsilon}$ has homological groups isomorphous to those of $X$, hence has same Betti numbers as $X$. Since we just involve finite sampled points and no message on the fundamental space $X$, creating a estimate of a proper range of $\varepsilon$ is impossible. To solve this problem, persistence was established by Edelsbrunner, Letscher, and Zomorodian [6] and exacted by Carlsson and Zomorodian [7]. Edelsbrunner, Letscher, and Zomorodian found the next fact. Whenever $\varepsilon \leq \varepsilon^{\prime}$, there exists a intrinsic inclusive relation of simplicial complexes $V R_{\varepsilon} \rightarrow V R_{\varepsilon^{\prime}}$, as the functorial character considered above, we get a linear transform $H_{k}\left(V R_{\varepsilon}\right) \rightarrow H_{k}\left(V R_{\varepsilon^{\prime}}\right)$ for any $k$. To research the topology of a space applying a finite point set sampled from it, we need understand all vector spaces $H_{k}\left(V R_{\varepsilon}\right)$, conjointly all the linear transforms discussed above. This economy is known as a persistent vector space, and the fact is proved in [7] that persistent vector spaces accept a category alike the category consequence for all finite dimension vector spaces, which claims that two vector spaces with equal dimension are isomorphous. For persistent vector spaces, it shows that affiliated to every persistent vector space, there exists an invariant known a barcode that is simply a finite set of intervals, and that any two persistent vector spaces having identical barcodes have an isomorphism between them. Big intervals of barcode denote real topological organization of an fundamental space, while small intervals are believed to be inadequate sampling. Fig. (1) shows a barcode of the Fig. (8).

In Fig. (1) we observe that there are one long line in dimension 0 , two in dimension 1 and no line in dimension 2 
which reflects the truth that the Fig. (8) has one connecting part and two holes in one-dimension.
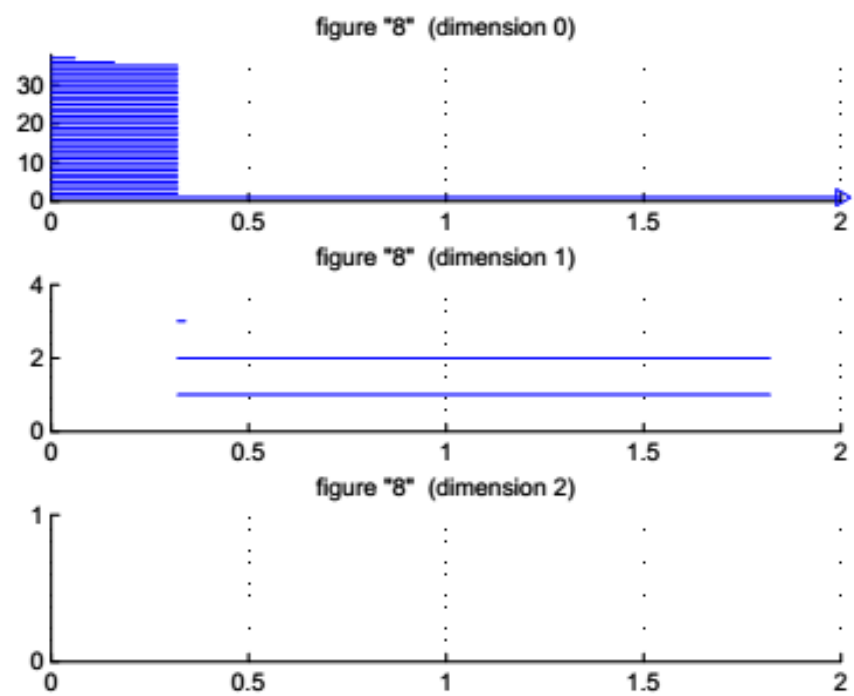

Fig. (1). The barcodes for the 40 points of Fig. (8).

Since $V R$ complexes may generate much higher simplexes in dimensions than that of the underlying space, in practice one applies lazy witness complex defined in [8].

Given a point cloud $P$, a landmark subset $L$, and a parameter $v \in N$, if $v=0$, let $m(q)=0$ for all $q \in P$. If $v>0$, let $m(q)$ be the distance $q$ to the $v$-th closest landmark point. The lazy witness complex $L W_{v}(P, L, \varepsilon)$ is defined as follows: (i) the vertex set is $L$; (ii) for vertices $a$ and $b$, edge $[a b]$ is in $L W_{v}(P, L, \varepsilon)$ if there exists a witness $q \in P$ such that

$\max \{d(a, q), d(b, q)\} \leq+m(q) ;$

(iii) a higher dimensional simplex is in $L W_{v}(P, L, \varepsilon)$ if all of its edges are.

The lazy witness complex relies upon a parameter $v$ $\in\{0,1,2\}$ which we choose to be $v=1$ : De Silva and Carlsson in [8] discovery $v=0$ to be basically ineffective, and $v$ $=2$ has malpractice of connecting each landmark point to anyhow another one at $R=0$. For more information and examples about lazy witness complexes, readers may refer to the papers $[8,9]$.

\section{SPACES OF RANGE IMAGE PATCHES}

One collects data sets of high-contrast $4 \times 4$ and $6 \times 6$ patches from range images. Each range data set include about $4 \times 10^{5}$ high-contrast log-range patches. These are drawn from the Brown database by Huang and Lee of approximately two hundreds range images [10], Fig. (2) are two samples.

The main spaces $M_{4}$ and $M_{6}$ are sets of $4 \times 4$ and $6 \times 6$ patches of high contrast constructed by 7 steps, which are analogous to the methods used in [3-5].

\section{RESULTS FOR $M^{4}(k, p)$ AND $M^{6}(k, p)$}

The $5 \times 5$ and $7 \times 7$ range patches have core subsets with topology of a circle were found by Adams and Carlsson in

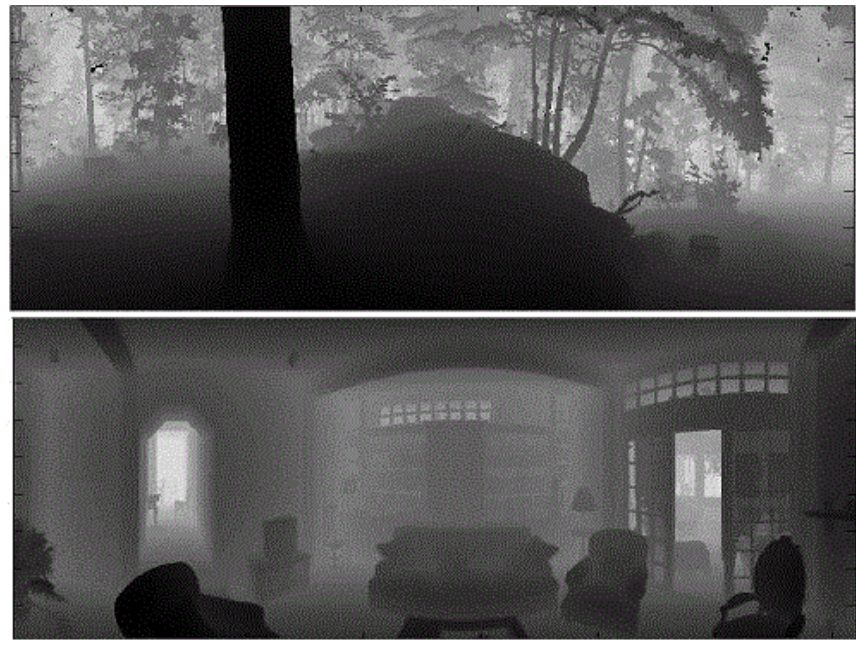

Fig. (2). Samples from the Brown range image database.
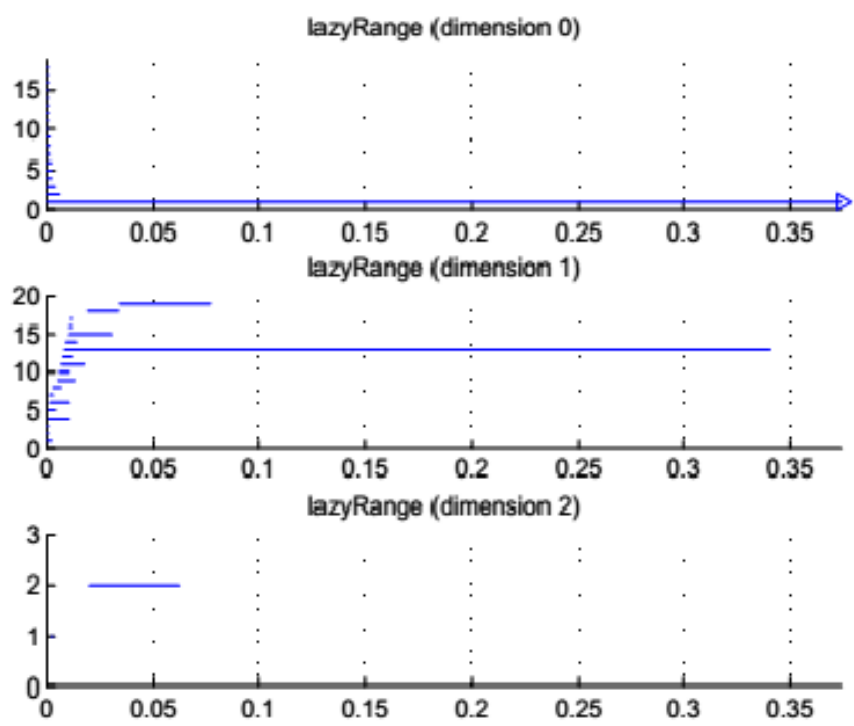

Fig. (3). Barcodes for $M^{4}(400,30)$.

[5]. To find the circle we need center (or core) subsets. We estimate the partial thickness of the space at a point $y$ by its the nearest neighbor. For $y \in X$ and $k>0$, let $\rho_{k}(y)=\left|y-y_{k}\right|$, here $y_{k}$ is the $k$-th nearest neighbor of $y$.

Larger $k$-values produce more global evaluations, whereas small $k$-values give local density evaluates. For a fixed $k$, we sort the points of $X$ by decreasing thickness, we pick the points denoted by $X(k, p)$ whose thickness are in peak $p$ percent. The center subset $X(k, p)$ may give important topological features, which may be lost for all the points of $X$.

Here we consider a core subset $M^{m}(k, p)$ of $\bar{M}$ for $m=$ 4 , 6. This subset represents appropriate core for proper values of two parameters $k$ and $p$.

The authors of the paper [3], observe that tall-contrast $3 \times 3$ range image blocks are thickly accumulated round the binary blocks. As found in [5], the topology of the core subset $M^{3}(300,30)$ is not clear. Considering the core subsets $M$ ${ }^{4}(400,30)$ and $M^{6}(300,30)$ for $4 \times 4$ and $6 \times 6$ patches, computing the barcode, an example of barcode plots for the 
center subsets $M^{4}(400,30)$ and $M^{6}(300,30)$ are given in Figs. (3) and (4), which shows that they have the topology of a circle, visible in Figs. (5) and (6). Selecting different 

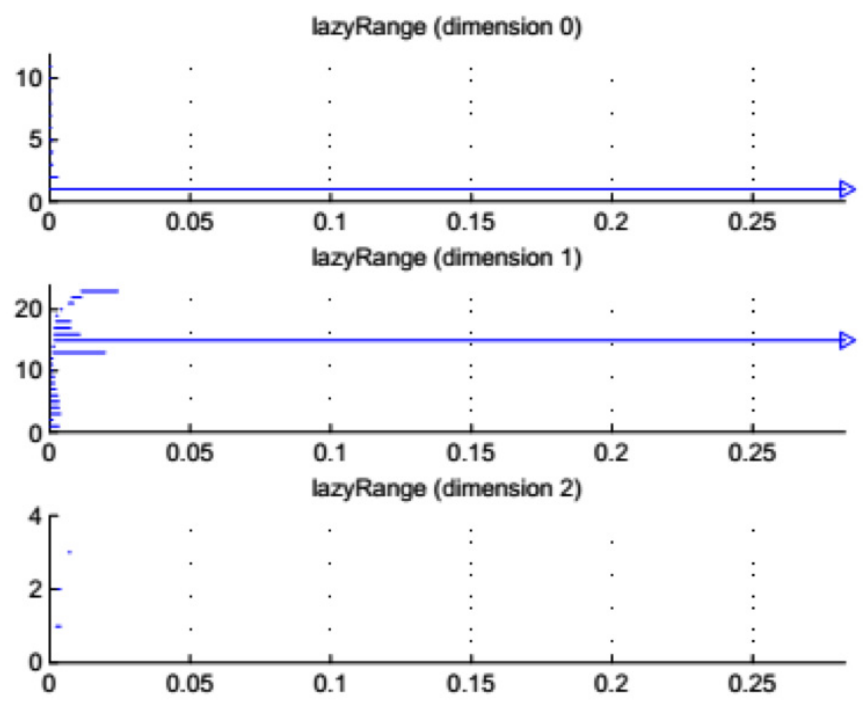

Fig. (4). Barcodes for $M^{6}(300,30)$.

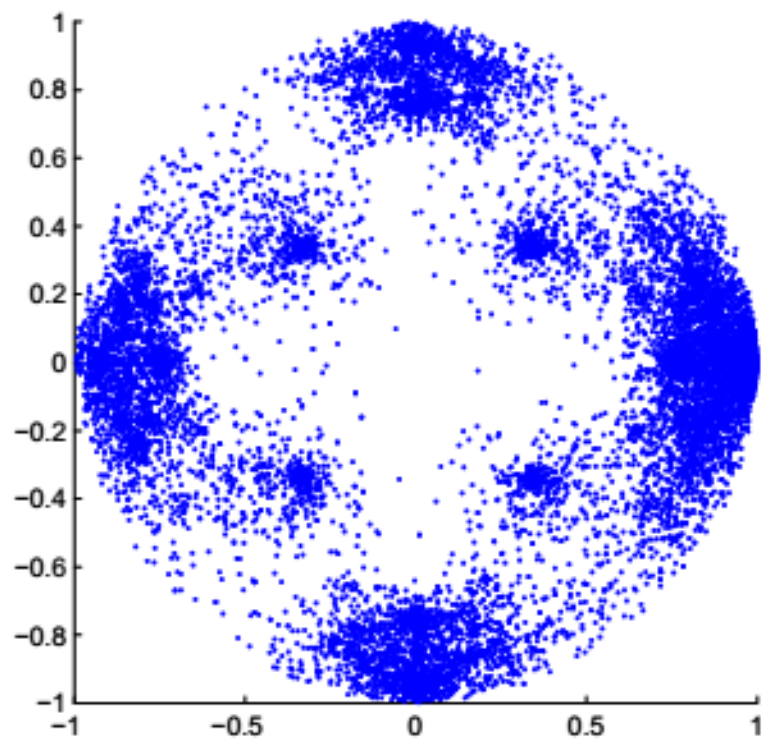

Fig. (5). Projection of $M^{4}(400,30)$ onto linear gradients.

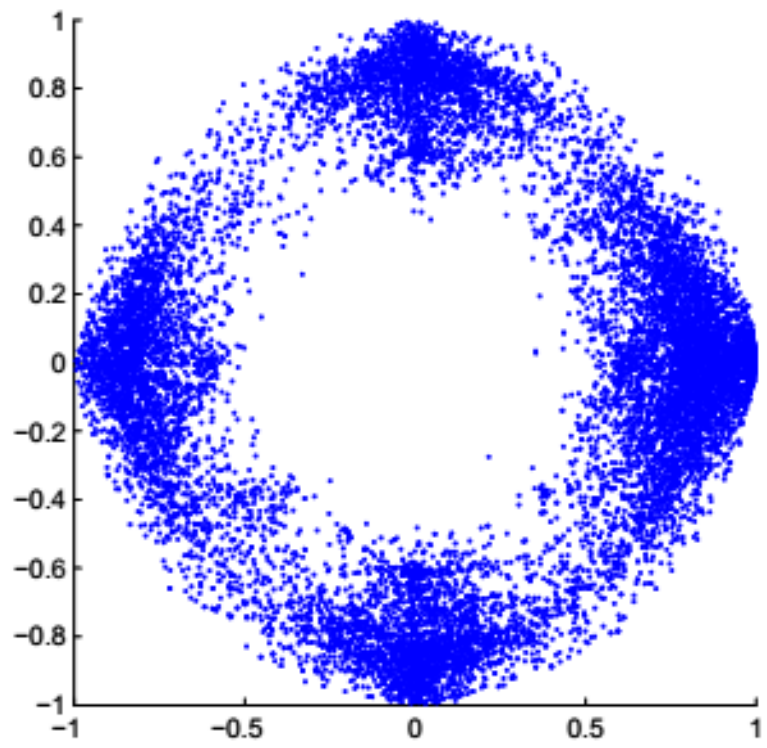

Fig. (6). Projection of $M^{6}(300,30)$ onto linear gradients.

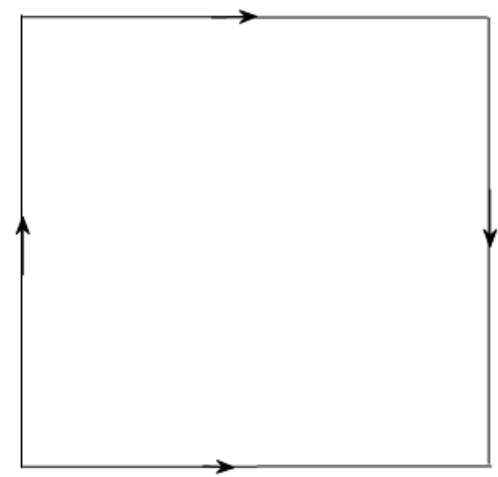

Fig. (7). Representation of Klein bottle as an identification space.

landmark points (from 35 to 60 ), we make many times trials on $M^{4}(400,30)$ and $M^{6}(300,30)$, and the results are very stable: in every experiment, the annular outline $\beta_{0}=\beta_{1}=1$ is found for nearly whole scope of $\varepsilon$ values, and other barcode intervals are very short $[11,12]$.

\section{KLEIN BOTTLE AND A SPACE OF MULTINOMI- ALS OF TWO VARIABLES}

We know that the Klein bottle be able to obtain by pasting a square in the plane as Fig. (7). To find the topological features of various subspaces of $M_{4}$ and $M_{6}$, we discuss another theoretical version of the Klein bottle. We will regard the $m \times m$ blocks as got though sampling a real valued smoothing function on the $x y$-plane at $m \times m$ mesh points. We now consider the space $\wp$, consisting of all polynomials with the shape $a_{2}\left(a_{1} x+b_{1} y\right)^{2}+b_{2}\left(a_{1} x+b_{1} y\right)$, where $a_{1}, b_{1}$, $a_{2}, \quad b_{2}$ are real numbers satisfying $\left(a_{1}, b_{1}\right) \in S^{1}$ and $\left(a_{2}, b_{2}\right) \in S^{1}$, here $S^{1}$ represents a unit loop.

The map $g: S^{1} \times S^{1} \mapsto \wp$ is defined by $\left(a_{1}, b_{1}, a_{2}, b_{2}\right)$ $\mapsto a_{2}\left(a_{1} x+b_{1} y\right)^{2}+b_{2}\left(a_{1} x+b_{1} y\right)$ [4]. It is easy to see that the map $g$ is onto, but not one to one, As the images of $\left(a_{1}, b_{1}, a_{2}, b_{2}\right)$ and $\left(-a_{1},-b_{1}, a_{2},-b_{2}\right)$ under $g$ are same. Thus, we have an equivalent relation $\left(a_{1}, b_{1}, a_{2}, b_{2}\right) \sim\left(-a_{1},-b_{1}, a_{2},-b_{2}\right)$. If $\left(a_{1}, b_{1}, a_{2}, b_{2}\right)$ is denoted as $(\cos \theta, \sin \theta, \cos \psi, \sin \psi), \theta$ and $\psi$ change at $[0$, $2 \pi]$, then the relation can be restated by $(\theta, \psi) \theta(\pi+\theta, 2 \pi-\psi)$. The space $\wp=\operatorname{im}(g)$ is homeomorphic to $g: S^{1} \times S^{1} /(\theta, \psi) \theta(\pi+\theta, 2 \pi-\psi)$, because of no other identifications created by $g$.

The ring surface can be represented by identifying a square with the opposite edges, that is a like as denotation of the Klein bottle, instead of identifying them with reversal orientation. The action of the mapping $g$ on the ring surface is shown in Fig. (8). The right half, indicated by $\mathrm{R}$ in the Fig. (8), is picked out by $g$ with the left-hand half, indicated by L. Each half is the description of the Klein bottle, hence the 
image under $g$ and Klein bottle are homeomorphic, and so are $\wp$ and Klein bottle [4].

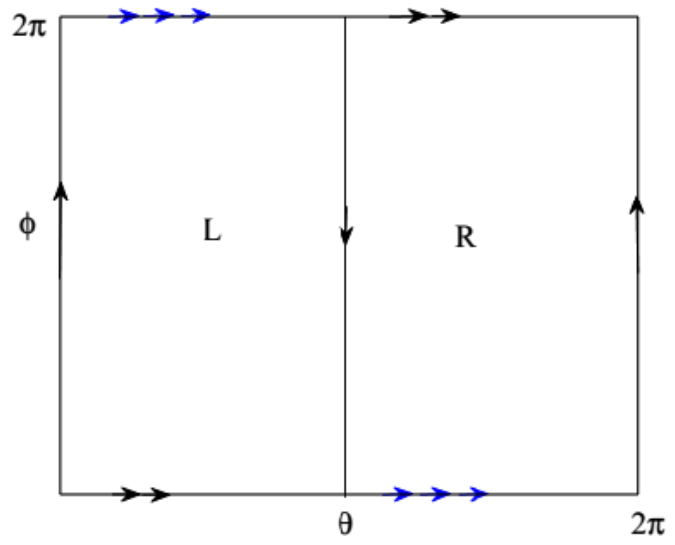

Fig. (8). The effect of the mapping $g$.
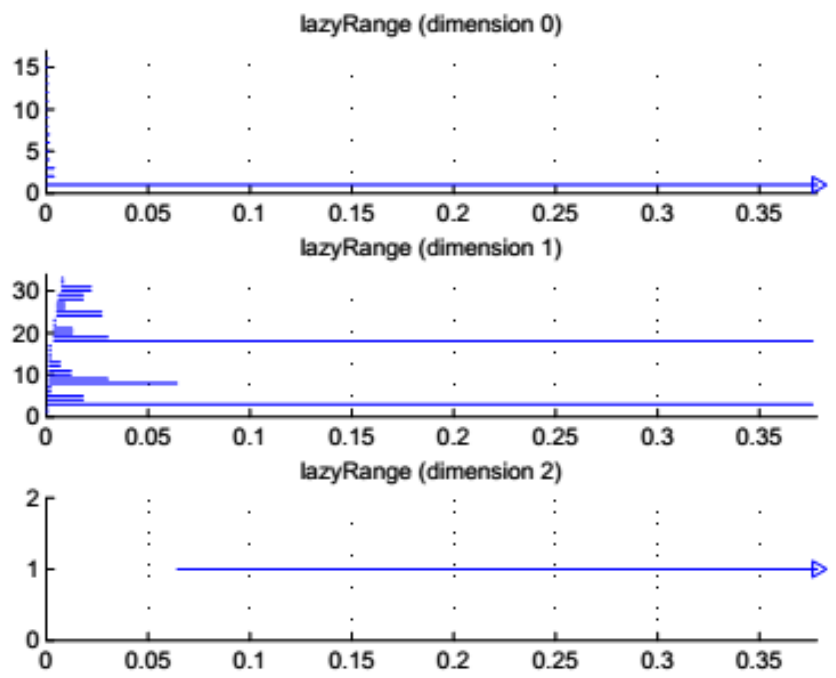

Fig. (9). Barcodes for $k 4 \_180$.

A map $h_{4}: \wp \mapsto S^{15}$ is defined by a composite of evaluating the multinomial at each point of the plane grid $H_{4}=\{-1$, $0,1,2\} \times\{-1,0,1,2\}$ minus the medium value and standardizing. A map $h_{6}: \wp \mapsto S^{35}$ by a composite of evaluating the polynomial at each point of the plane grid $H_{6}=\{-2,-1$, $0,1,2,3\} \times\{-2,-1,0,1,2,3\}$ minus the medium value and normalizing.

As the proof in [4], the image $\operatorname{im}\left(h_{m}\right)(m=4,6)$ and the Klein bottle are homeomorphic.

In order to inserted the Klein bottle till a unit sphere, firstly, we uniformly select 180 points $\left(\left\{x_{1}, \ldots, x_{180}\right\}\right)$ from the unite circle, all tuples $\left(x_{i}, x_{j}\right)$ form a point set on the torus $S^{1} \times S^{1}$. Then, we map all of the 32400 points into $S^{15}$ and $S$ 35 by maps $h_{4}{ }^{\circ} \mathrm{g}$ and $h_{6}{ }^{\circ} \mathrm{g}$, the image of each map is denoted by $k 4 \_180$ and $k 6 \_180$ respectively. Figs. (9) and (10) give the PLEX results for the homology of the spaces $k 4 \_180$, $k 6 \_180$. Each of the Figures shows $\beta_{0}=1, \beta_{1}=2$ and $\beta_{2}=1$, that are mod two betti numbers of the Klein bottle. Hence we have that $k 4 \_180(k 6180)$ is a reasonable approximation of the Klein bottle in $S^{15}\left(S^{35}\right)$.
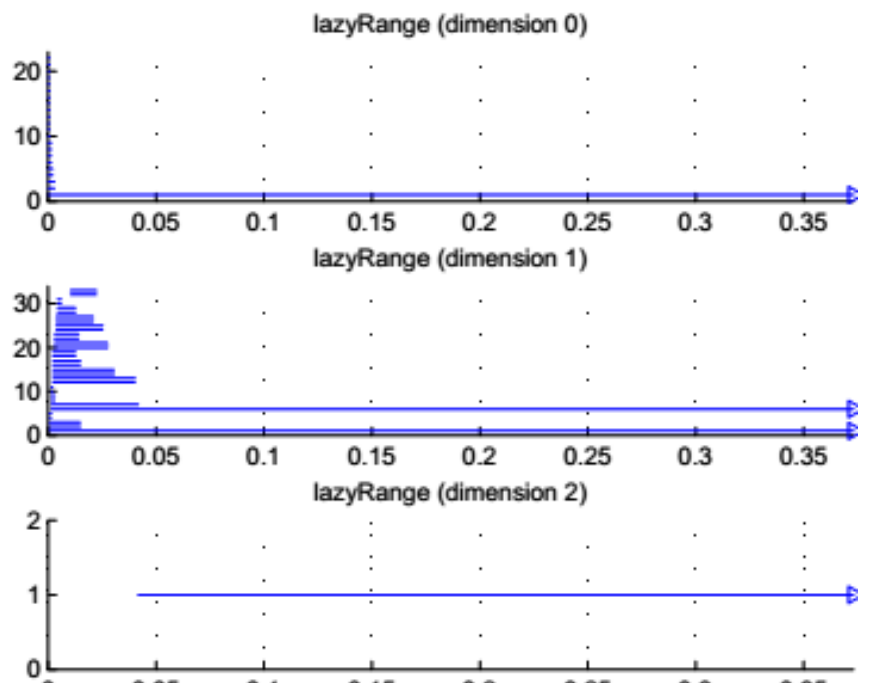

Fig. (10). Barcodes for $k 6 \_180$.
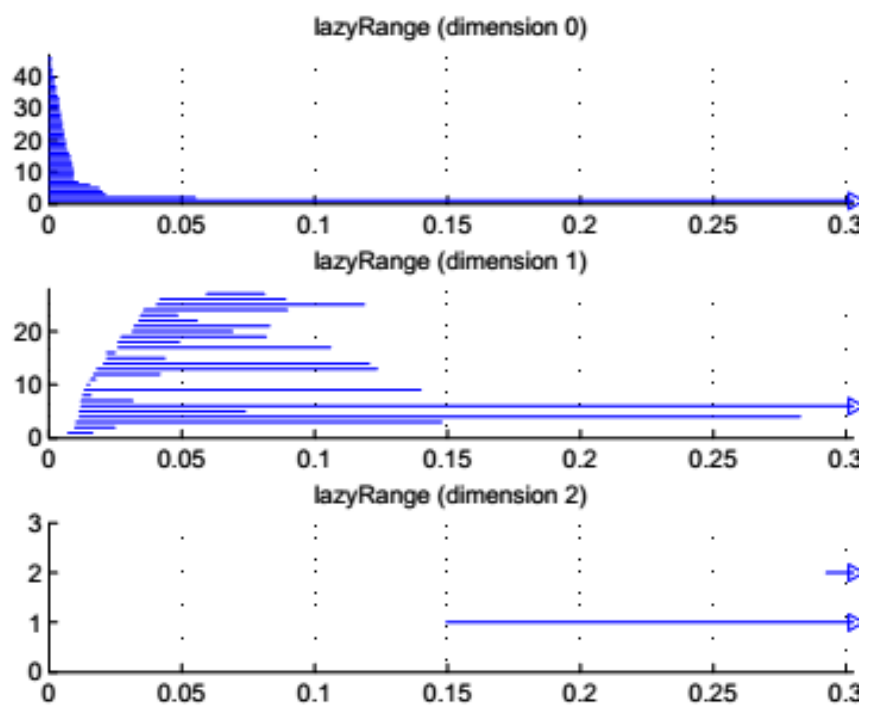

Fig. (11). PLEX results for $X k(4,180,3)$. 


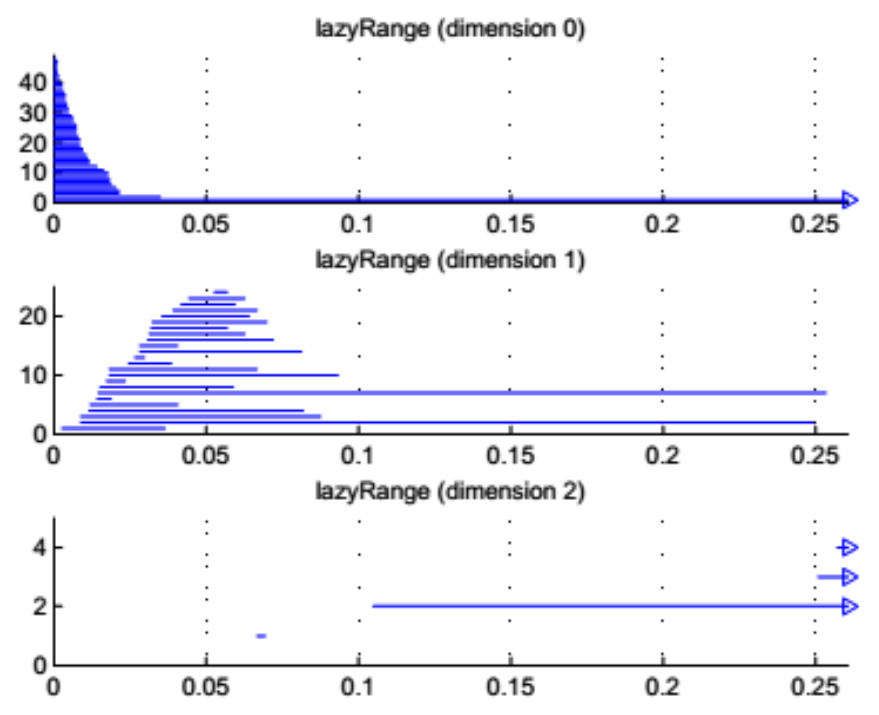

Fig. (12). PLEX results for $X k(6,180,7)$.

\section{RESULTS FOR $M^{4}$ AND $M^{6}$}

We know that $S^{15}$ and $S^{35}$ has subspaces $k 4 \_180$ and $k 6$ 180 separately, their homology is same as of a Klein bottle, by using them subspaces of $M_{4}$ and $M_{6}$ can be found, and the subspaces have the topology of the Klein bottle shown by experimentation. We depict how to build subspaces of $M_{4}$ and $M_{6}$ as follows.

For any point $x$ of $k 4 \_180$ one calculate the Euclidean distance from $x$ to every point of $M_{4}$, then pick $m$ closest points to the point $x$. The subspace of $M_{4}$ is obtained by collecting all $m$ closest points to any $x \in \mathrm{k} 4 \_180$, denoted by $X k(4,180, m)$. The subspace $X k(6,180, m)$ of $M_{6}$ is obtained by a similar way.

To detect $M_{4}$ and $M_{6}$ have subspaces with the topology of the Klein bottle, we use subspaces $X k(4,180,3)$ and $X k(6$, $180,7)$. Fig. (11) shows that $X k(4,180,3)$ has the homology of the Klein bottle from the parameter values 0.15 to 0.28 . Fig. (12) shows that $X k(6,180,7)$ has the homology of the Klein bottle from the parameter values 0.11 to 0.25 . We make more than 150 experiments on $X k(4,180,3)$ and $X k$ $(6,180,7)$ by choosing different landmark points, all PLEX results indicate that $\beta_{0}=1, \beta_{1}=2$ and $\beta_{2}=1$ at some interval of $\varepsilon$ values, hence the results are very stable.

\section{CONCLUSION}

In this paper we utilize persistent homology to research qualitative topological analysis of spaces of $4 \times 4$ and $6 \times 6$ range patches. The greatest advantage of the topological methods is that it admits one to discover non-linear confor- mations in the data that will be extremely difficult to discover by statistical skills. We certify that the spaces of highcontrast $4 \times 4$ and $6 \times 6$ patches have core subsets modeled as the primary circle. By the relation among the spaces of range image blocks and the space of multinomials with 2-variables, it is shown that there exist subspaces of $M_{4}, M_{6}$, whose homology is that of a Klein bottle. We conformed our findings by applying the same methods to the different sizes patches. These express that the behavior of optical patches and range patches may be very similar, and propose that using the Klein bottle for the aims of image compression could really be popularize to the case of range images.

\section{CONFLICT OF INTEREST}

The authors confirm that this article content has no conflict of interest.

\section{ACKNOWLEDGEMENTS}

This work was supported by the National Natural Science Foundation of China (No.61471409).

\section{REFERENCES}

[1] U. Grenander, and A. Srivastava, "Probability models for clutter in natural images", IEEE Transactions on PAMI, vol. 23, pp. 424429, 2001.

[2] B.A. Olshausen, and D.J. Field, "Natural image statistics and efficient coding", Network: Computation in Neural Systems, vol. 7, pp. 333-339, 1996.

[3] A. B. Lee, K. S. Pedersen, and D. Mumford, "The non-linear statistics of high-contrast patches in natural images", International Journal of Computer Vision, vol. 54, pp. 83-103, 2003.

[4] G. Carlsson, T. Ishkhanov, V. De Silva, and A. Zomorodian, "On the local behavior of spaces of natural images", International Journal of Computer Vision, vol. 76, pp. 1-12, 2008.

[5] H. Adams and G. Carlsson, "On the nonlinear statistics of range image patches”, SIAM Journal of Imaging Sciences, vol. 2, pp. $110-117,2009$

[6] H. Edelsbrunner, D. Letscher, and A. Zomorodian, "Topological persistence and simplification", Discrete Computational Geometry, vol. 28 , pp. 511-533, 2002.

[7] A. Zomorodian, and G. Carlsson, "Computing persistent homology”, Discrete Computational Geometry, vol. 33, pp. 249-274, 2005.

[8] V. De Silva, and G. Carlsson, Topological estimation using witness complexes, Symposium on Point-Based Graphics, 2004

[9] H. Adams, and A. Tausz, Javaplex tutorial, http://javaplex. googlecode.com/svn/trunk/reports/javaplex tutorial/javaplex tutorial.pdf.

[10] J. Huang, A. Lee, and D. Mumford, "Statistics of range images", In Proceedings of IEEE Conference on Computer Vision and Pattern Recognition, Hilton Head Island, SC, vol. 1, pp. 324-333, 2000.

[11] A. Hatcher, Algebraic Topology, Cambridge University Press: USA, 2002.

[12] G. Carlsson, "Topology and data", Bulletin (New Series) of the American Mathematical Society, vol. 46, pp. 255-308, 2009. 